\title{
Immunosuppressant therapy successfully improved regorafenib-induced severe hepatic injury in a patient with metastatic gastrointestinal stromal tumor: A case report
}

\author{
MIYUKI KUWAYAMA ${ }^{1}$, KEITA UCHINO ${ }^{1}$, KOTOE TAKAYOSHI ${ }^{1,2}$, MASATO KOMODA $^{1}$, MOTOYUKI KOHJIMA ${ }^{3}$, \\ MAKOTO NAKAMUTA ${ }^{3}$, SEIYA MOMOSAKI ${ }^{4}$, HITOSHI KUSABA ${ }^{2}$, KOICHI AKASHI $^{2}$ and EISHI BABA ${ }^{5}$ \\ ${ }^{1}$ Department of Medical Oncology, Clinical Research Institute, National Hospital Organization Kyushu Medical Center, \\ Fukuoka 810-0065; ${ }^{2}$ Department of Hematology and Oncology, Kyushu University Hospital, Fukuoka 812-8582; \\ Departments of ${ }^{3}$ Gastroenterology and ${ }^{4}$ Pathology, National Hospital Organization Kyushu Medical Center, Fukuoka 810-0065; \\ ${ }^{5}$ Department of Comprehensive Clinical Oncology, Faculty of Medical Sciences, Kyushu University, Fukuoka 812-8582, Japan
}

Received November 21, 2014; Accepted September 18, 2015

DOI: $10.3892 / \mathrm{ol} .2015 .3853$

\begin{abstract}
A 75-year-old man diagnosed with ileal gastrointestinal tumor with peritoneal dissemination was subjected to salvage treatment with regorafenib at $120 \mathrm{mg} /$ day. Following the initiation of the treatment, liver dysfunction appeared on day 28, and continued to worsen despite termination of the treatment. Since no increase in the levels of serum immunoglobulins of the patient was observed, and negative results were obtained for the analysis of viral markers and autoantibodies, a diagnosis of regorafenib-induced hepatitis was suggested. In consequence, the patient received steroid pulse therapy and continuous administration of prednisolone, without sufficient improvement. Liver biopsy revealed interface hepatitis with prominent plasma cell infiltration, suggesting regorafenib-induced autoimmune hepatitis. The patient was then administered azathioprine and prednisolone, which improved the hepatic injury. The present case represents the first report of successful treatment of regorafenib-induced severe hepatic injury by the use of an immunosuppressant.
\end{abstract}

\section{Introduction}

Gastrointestinal stromal tumors (GISTs) are common sarcomas arising in the gastrointestinal tract (1). Advanced GISTs are generally treated using a combination of surgical resection and chemotherapies (2). The recent development of kinase

Correspondence to: Professor Eishi Baba, Department of Comprehensive Clinical Oncology, Faculty of Medical Sciences, Kyushu University, 3-1-1 Maidashi, Higashi-ku, Fukuoka 812-8582, Japan

E-mail: e-baba@c-oncology.med.kyushu-u.ac.jp

Key words: gastrointestinal stromal tumor, regorafenib, liver injury, drug-induced autoimmune hepatitis, immunosuppressant inhibitors, including imatinib mesylate, sunitinib malate and regorafenib, has markedly improved the clinical outcomes of patients with advanced metastatic GIST (2).

Regorafenib is an oral multikinase inhibitor that interferes with multiple signaling pathways involved in the proliferation and survival of tumor cells, including those mediating angiogenesis, oncogenesis and maintenance of the tumor microenvironment (3). A previous phase III clinical study, known as the GRID study, examined the efficacy of regorafenib over placebo for standard therapy-refractory metastatic GISTs, and demonstrated that patients treated with regorafenib experienced a superior progression-free survival, compared with the placebo group (3). In addition, regorafenib was also observed to be effective for the treatment of advanced colorectal cancers (CRCs) in a global phase III study of 760 patients (the CORRECT study) (4). Thus, regorafenib is currently used worldwide as an anticancer drug (4).

However, treatment with regorafenib may lead to adverse events. In the CORRECT study (4), toxicities of common terminology criteria of adverse events grade $\geq 3$ appeared in $59.8 \%$ of the patients who received regorafenib. While hypertension, hand-foot skin reaction and diarrhea were observed in 23.5, 19.7 and $5.3 \%$ of patients, respectively, grade $\geq 3$ hyperbilirubinemia was observed in $2 \%$ patients, and no other adverse events associated with liver dysfunction were reported. In the GRID study (3), liver dysfunction was reported among the drug-related adverse events observed in $\leq 10 \%$ of patients, and frequent increased levels of serum transaminase were reported in sub-analyses of Japanese patients in the CORRECT study (5). Liver dysfunction occurring in association with administration of regorafenib has been previously recognized as drug-induced hepatitis (DIH) (6). Patients suffering liver dysfunction related to regorafenib often discontinue the treatment, and are subsequently administered liver-protecting agents (7). Although treatment with steroids and plasmapheresis has previously been employed for patients with DIH in a severe state, fatal results have occasionally occurred.

Drug-induced autoimmune hepatitis (DIAIH) is one of the categories of autoimmune hepatitis (AIH) (7). The damage 


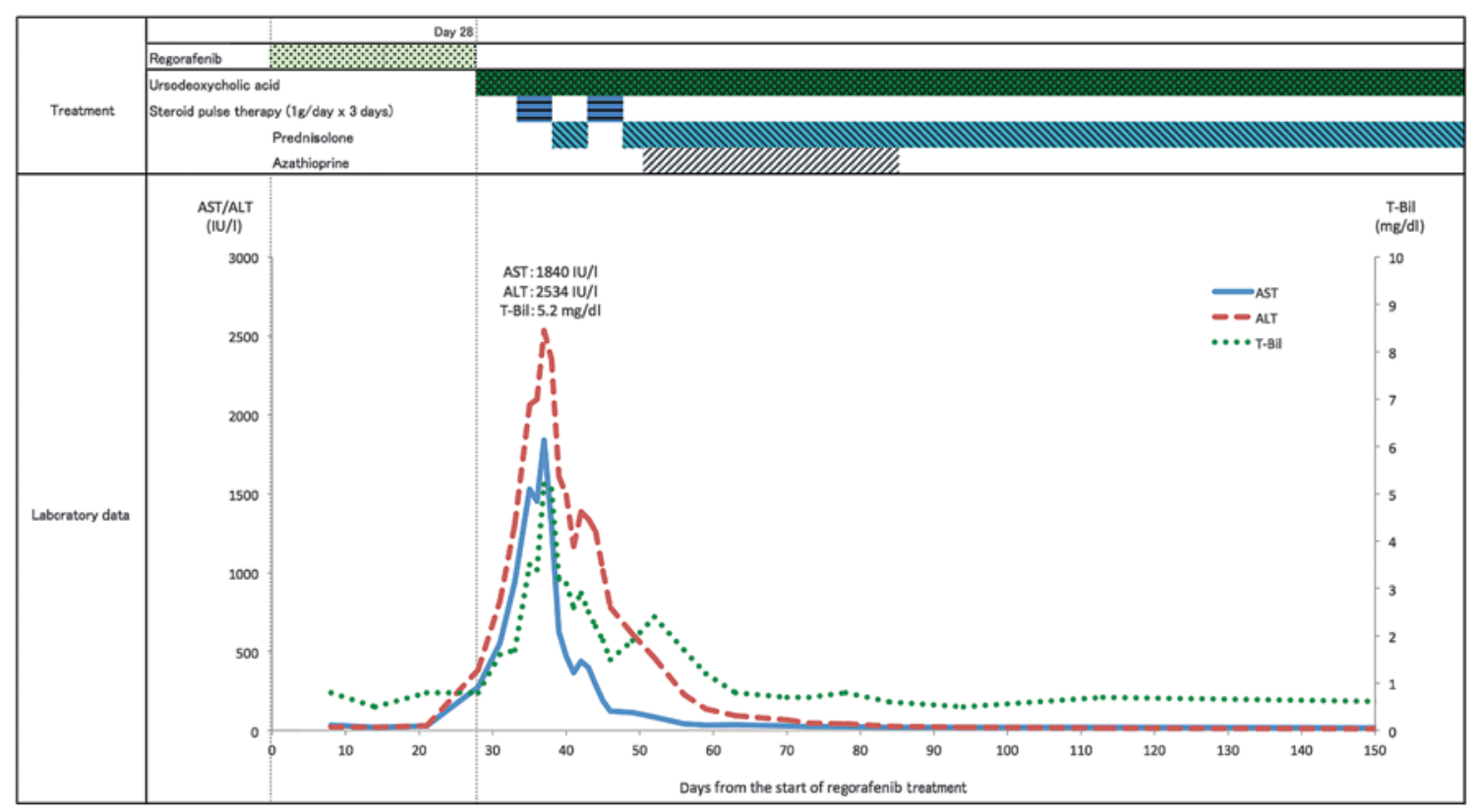

Figure 1. Clinical course and variation in the serum levels of ALT, AST and T-Bil. ALT, alanine aminotransferase; AST, aspartate aminotransferase; T-Bil, total bilirubin.

to hepatocytes observed in DIAIH is considered to develop through autoimmune reactions associated with a certain drug, while DIH represents direct injury to hepatocytes by a causative drug (7). DIAIH and AIH share the histological features of hepatitis in association with the infiltration of plasma cells (7). The frequency of DIAIH among classical AIH has been estimated to be $9 \%$, but a limited number of drugs such as minocycline and nitrofurantoin have been reported to often induce DIAIH (8). In the present case report, the first description of DIAIH possibly caused by regorafenib, and its successful treatment with the immunosuppressant drug azathioprine, is described.

\section{Case report}

Anemia was identified in a 75-year-old man visiting his local doctor for treatment of hypercholesterolemia and periodic examination in September 2011. The patient had undergone appendectomy $\sim 50$ years earlier, and presented no history of drug allergy, although the patient had been smoking 20 cigarettes/day and drinking $40 \mathrm{~g}$ of alcohol/day during 50 years. Computed tomography $(\mathrm{CT})$ revealed an intrapelvic tumor of $10 \mathrm{~cm}$ in diameter and several small nodules of $\sim 1 \mathrm{~cm}$ in diameter in the abdominal cavity. Gastrointestinal endoscopy did not reveal any specific findings.

In September 2011, the patient was admitted to the National Hospital Organization Kyushu Medical Center (Fukuoka, Japan), and surgical resection of the intrapelvic tumor and partial ileectomy were then performed. Macroscopically, the tumor was $10.5 \mathrm{~cm}$ in diameter and limited to the muscular layer of the terminal ileum. Histological examination revealed spindle-shaped atypical cells proliferating in fascicular form. Immunohistochemically, the tumor cells were positive for c-kit and cluster of differentiation 34, partially positive for $\alpha$-smooth muscle actin (SMA), and negative for desmin and S-100. Therefore, the postoperative diagnosis was ileal GIST with peritoneal dissemination, representing a high-risk status. Consequently, administration of imatinib mesylate at $400 \mathrm{mg} /$ day was started in December 2012. However, in January 2013, high fever appeared, and radiological examination detected bilateral interstitial pneumonia. Imatinib therapy was subsequently terminated, and the interstitial pneumonia immediately improved.

During the observational period following imatinib therapy, the peritoneal tumors gradually enlarged. Therefore, oral administration of regorafenib at $120 \mathrm{mg}$ /day was initiated in October 2013 (Fig. 1). However, the therapy was discontinued 9 days later, due to the appearance of fever, and restarted on day 15 , following complete recovery. Increased levels of serum transaminases, including alanine aminotransferase (ALT) (274 IU/l) and aspartate aminotransferase (AST) (385 IU/l), and total bilirubin (T-Bil) $(1.1 \mathrm{mg} / \mathrm{dl})$ were detected on day 28 . In consequence, the treatment with regorafenib was terminated. However, the laboratory findings continued to worsen despite suspending the treatment, and the patient was readmitted to the hospital 31 days later. Further increase in the levels of T-Bil (5.2 mg/dl), AST (1,840 IU/l) and ALT (2,534 IU/l) were observed on day 37. CT scan on day 35 revealed no significant lesions in the liver and a marked improvement of the peritoneal disseminations. No other adverse events were noticed during this period. Laboratory analyses revealed no increase in the levels of serum immunoglobulin ( $\mathrm{Ig}$ ), and negative results were obtained for viral markers of hepatitis B and C, antinuclear antibody and $\alpha$-SMA, suggesting that viral hepatitis and $\mathrm{AIH}$ were unlikely.

In consequence, regorafenib-induced hepatitis was diagnosed, and steroid pulse therapy (1,000 mg/day) was initiated at day 37. Continuous administration of prednisolone at $40 \mathrm{mg} /$ day was started at day 40, but limited improvement was observed. Based on the histological examination of a specimen 


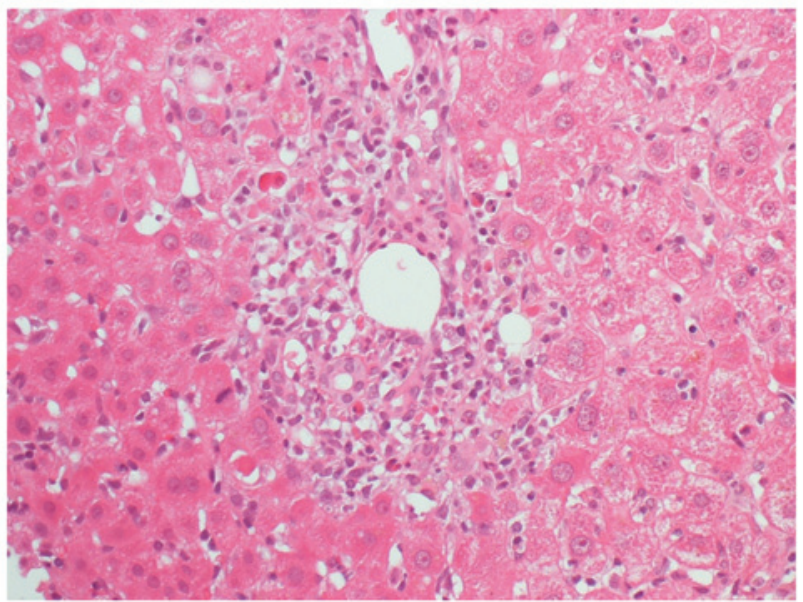

Figure 2. Histological examination of liver tissue obtained by biopsy on day 50 post-initiation of regorafenib therapy. The sample slide was stained with hematoxylin and eosin, and observed by light microscopy at high power (magnification, $\mathrm{x} 400$ ).

derived from liver biopsy conducted at day 50, which exhibited interface hepatitis with plasma cell infiltration (Fig. 2), regorafenib-induced autoimmune hepatitis was suggested. Administration of prednisolone (30 mg/day) in addition to the immunosuppressant drug azathioprine (50 mg/day) markedly ameliorated the liver dysfunction, and the patient experienced complete recovery within one month. No evidence of progression of DIAIH following withdrawal of azathioprine was observed on day 91 and also with reduced dose of prednisolone ( $5 \mathrm{mg} /$ day) was observed on day 150 . Upon recovery from liver dysfunction, chemotherapy was terminated, and the patient was under follow-up for 14 months. During this period, the peritoneal GISTs exhibited a moderate growth, although the patient did not experience any specific symptoms.

\section{Discussion}

The patient of the present case report exhibited acute-onset hepatic injury following the initiation of regorafenib therapy. The clinical features and histological findings from liver tissue suggested DIAIH. DIAIH is one of the categories of AIH, and shares similar characteristics with it, including predominant incidence among women, presence of autoantibodies, increased levels of serum $\mathrm{IgG}$, and infiltration of lymphocytes and plasma cells into liver tissue (9). These features of AIH differ from those observed in the patient of the present case report. Thus, the gender of the patient (man), his lack of autoantibodies and the normal levels of serum IgG displayed by the patient were suggestive of DIH. Although autoantibodies are considered to be suitable markers for distinguishing DIAIH from other forms of DIH, drugs such as nitrofurantoin and methyldopa, known to induce DIAIH, may not trigger autoantibody production in $20-40 \%$ of patients with DIAIH attributable to these agents, according to previous reports (10). Furthermore, histological examination of the liver in the present case clearly revealed specific features of DIAIH, and the prominent effectiveness of the immunosuppressant therapy administered to the patient further supported the diagnosis of DIAIH. In addition, the absence of a relapse in hepatitis following the withdrawal of immunosuppressants and tapering of prednisolone to a dose of $5 \mathrm{mg} /$ day was also consistent with DIAIH.

In previous clinical studies examining the efficacy of regorafenib for the treatment of GIST and CRC, low incidences of adverse events in terms of liver function were reported $(3,4)$. However, a more frequent appearance of adverse events related to liver dysfunction has been recognized among Asian populations, particularly in Japanese patients (5). High levels of transaminase, classed as grade $\geq 3$, were more frequently observed in the Japanese subpopulation, compared with the whole cohort in the CORRECT study (5), while in the other CORRECT study (4), a 62-year-old Asian man suffered grade 5 drug-induced liver dysfunction. A previous retrospective study of 32 cases of metastatic CRC revealed that 19 and $16 \%$ of patients exhibited increased levels of AST and ALT of grade $\geq 3$, respectively, and 1 patient succumbed to liver dysfunction under salvage-line regorafenib monotherapy (11). In a previous prospective phase III study (4), intensive supportive care, including plasmapheresis, was unable to rescue a proportion of patients with regorafenib-related liver dysfunction. However, the etiologies of liver dysfunction in association with regorafenib were not clearly described in these studies. Generally, in previous cases of patients diagnosed with regorafenib-induced hepatitis, the administration of the drug was discontinued, and the patients subsequently received liver-protecting agents (6). Considering the clinical courses of the population who suffer regorafenib-induced liver dysfunction, the establishment of appropriate methods of diagnosis and specific therapies for DIAIH is required.

In the present case, the reasons leading to the development of DIAIH in association with regorafenib remain unclear. Possible risk factors for DIAIH due to exposure to certain drugs have been previously proposed, particularly in terms of the patient's background and the characteristics of the causative agents (7). Patient's age, female gender and alcohol intake have been identified as risk factors for DIAIH (7). In addition, genetic predispositions to DIAIH have been reported, including human leukocyte antigen B8 haplotype for clometacin-induced AIH and DRB1*03 genotype for isoniazid-induced $\mathrm{AIH}$, suggesting that certain genetic backgrounds may induce specific forms of DIAIH (12). While the majority of cases of DIAIH are considered to be caused by minocycline and nitrofurantoin, a previous report has described one case of AIH induced by imatinib, a molecular targeting agent usually employed for the treatment of malignant neoplasms (13).

Current therapeutic strategies for DIAIH consist of discontinuation of the causative agent and subsequent administration of liver-protecting agents, steroids and immunosuppressants. The use of immunosuppressants for the treatment of DIAIH is based on the pathogenesis of this condition, since the dominant mechanism of hepatocyte injury depends on the infiltrating immune cells (7). In the present case, regorafenib-induced severe liver injury appeared refractory to prednisolone therapy, and responded to the subsequent azathioprine therapy.

In conclusion, the present case report offers the first description of possible regorafenib-induced $\mathrm{AIH}$ in a patient with metastatic GIST. Based on the findings of the present study, it can be proposed that liver biopsies for patients with refractory drug-induced liver dysfunction may aid to achieving the appropriate diagnosis and providing valuable information 
for selecting the adequate therapy. Further exploration of the actual incidence of regorafenib-induced liver injuries such as AIH among patients with CRC and GIST, and clarification of the molecular mechanisms involved in the development of $\mathrm{DIH}$, is required.

\section{Acknowledgements}

The authors would like to thank the medical staff of the National Hospital Organization Kyushu Medical Center (Fukuoka, Japan), who contributed to the treatment of the patient.

\section{References}

1. Hirota S, Isozaki K, Moriyama Y, Hashimoto K, Nishida T, Ishiguro S, Kawano K, Hanada M, Kurata A, Takeda M, et al: Gain-of-function mutations of c-kit in human gastrointestinal stromal tumors. Science 279: 577-580, 1998

2. Demetri GD, von Mehren M, Antonescu CR, DeMatteo RP, Ganjoo KN, Maki RG, Pisters PW, Raut CP, Riedel RF, Schuetze S, et al: NCCN Task Force report: Update on the management of patients with gastrointestinal stromal tumors. Natl Compr Canc Netw 8. Suppl 2: S1-S41, 2010.

3. Demetri GD, Reichardt P, Kang YK, Blay JY, Rutkowski P, Gelderblom H, Hohenberger P, Leahy M, von Mehren M, Joensuu H, et al; GRID study investigators: Efficacy and safety of regorafenib for advanced gastrointestinal stromal tumours after failure of imatinib and sunitinib (GRID): An international, multicentre, randomised, placebo-controlled, phase 3 trial. Lancet 381: 295-302, 2013.
4. Grothey A, Van Cutsem E, Sobrero A, Siena S, Falcone A, Ychou M, Humblet Y, Bouché O, Mineur L, Barone C, et al; CORRECT Study Group: Regorafenib monotherapy for previously treated metastatic colorectal cancer (CORRECT): An international, multicentre, randomised, placebo-controlled, phase 3 trial. Lancet 381: 303-312, 2013.

5. Yamada Y, Yoshino T, Komatsu Y Yamazaki K, Tsuji A, Ura T, Grothey A, Van Cutsem E, Wagner A and Ohtsu A: Safety and efficacy of regorafenib in Japanese patients with metastatic colorectal cancer: A subgroup analysis of the phase III CORRECT trial. Ann Oncol 24 (Suppl 9): ix31-ix65, 2013.

6. Leise MD, Poterucha JJ and Talwalkar JA: Drug-induced liver injury. Mayo Clin Proc 89: 95-106, 2014.

7. Czaja AJ: Drug-induced autoimmune-like hepatitis. Dig Dis Sci 56: 958-976, 2011

8. Björnsson E, Talwalkar J, Treeprasertsuk S, Kamath PS, Takahashi N, Sanderson S, Neuhauser M and Lindor K: Drug-induced autoimmune hepatitis: Clinical characteristics and prognosis. Hepatology 51: 2040-2048, 2010.

9. Krawitt EL: Autoimmune hepatitis. N Engl J Med 354: 54-66, 2006.

10. Lewis JH and Zimmerman HJ: Liver diseases. In: Auto- immune Liver Diseases. Krawitt EL, Wiesner RH and Nishioka K (eds). 2nd edition. Elsevier, Amsterdam, Netherlands, pp 627-649, 1998.

11. Hirano G, Makiyama A, Makiyama C, Esaki T, Oda H, Uchino K, Komoda M, Tanaka R, Matsushita Y, Mitsugi K, et al: Reduced-dose of salvage-line regorafenib monotherapy for metastatic colorectal cancer in Japan. Anticancer Res 35: 371-377, 2015.

12. Russmann S, Jetter A and Kullak-Ublick GA: Pharmacogenetics of drug-induced liver injury. Hepatology 52: 748-761, 2010.

13. Aliberti S, Grignani G, Allione P, Fizzotti M, Galatola G, Pisacane A and Aglietta M: An acute hepatitis resembling autoimmune hepatitis occurring during imatinib therapy in a gastrointestinal stromal tumor patient. Am J Clin Oncol 32: 640-641, 2009 